\title{
Gallbladder Neuroendocrine Tumor G1
}

National Cancer Institute

\section{Source}

National Cancer Institute. Gallbladder Neuroendocrine Tumor G1. NCI Thesaurus. Code C96924.

A well differentiated, low grade neuroendocrine tumor (carcinoid tumor) that arises from the gallbladder. The mitotic count is less than 2 per $10 \mathrm{HPF}$ and/or the Ki67 index is equal to or less than 2 percent. 